\title{
FATORES QUE CONTRIBUEM PARA A GRAVIDEZ NÃO PLANEJADA EM USUÁRIAS DO PROGRAMA DE PLANEJAMENTO FAMILIAR
}

\section{FACTORS THAT CONTRIBUTE TO UNPLANNED PREGNANCY IN USERS OF THE FAMILY PLANNING PROGRAM}

\author{
Rosemeire de Jesus Santos
}

Faculdade Zacarias de Góes

\begin{abstract}
To analyze the knowledge produced in the scientific literature on factors that contribute to unplanned pregnancy in users of the family planning program. An integrative literature review was carried out by searching the LILACS, BDENF, MEDLINE and SCIELO databases, between 2010 and 2018, the descriptors used were: "family planning" and "unplanned pregnancy", after applying the inclusion criteria, eight articles were selected for discussion. The factors associated with the occurrence of unplanned pregnancies are interconnected, the low adherence to the family planning program, mainly by adolescents, women with low education, black or brown skin color, multigravidae, who reported having a partner and having low family income. The interrelationship between the various factors associated with unplanned pregnancy demonstrated the need to implement public policies aimed at women in different contexts.
\end{abstract}

Key words: Pregnancy, Unplanned; Family planning; Contraception; Family Planning Services; Family Planning Policy.

\section{Resumo}

Analisar o conhecimento produzido na literatura científica sobre fatores que contribuem para a gravidez não planejada em usuárias do programa de planejamento familiar. Foi realizada uma revisão de literatura integrativa por meio de pesquisa nas bases de dados LILACS, BDENF, MEDLINE e SCIELO, no intervalo temporal de 2010 a 2018, os descritores utilizados foram: "planejamento familiar" e "gravidez não planejada", após aplicação dos critérios de inclusão, oito artigos foram selecionados para a discussão. Os fatores associados à ocorrência de gestação não planejada estão interligados, a baixa adesão ao programa de planejamento familiar, principalmente por adolescentes, mulheres com baixa escolaridade, de cor de pele preta ou parda, multigestas, que relataram ter companheiro e possuir baixa renda familiar. A inter-relação entre os diversos fatores associados à gravidez não planejada demostrou a necessidade de efetivação de políticas públicas voltadas para mulheres inseridas em diversos contextos.

Palavras-chave: Gravidez não planejada; Planejamento Familiar; Anticoncepção; Serviços de Planejamento Familiar; Política de Planejamento Familiar. 


\section{Introdução}

O programa de planejamento familiar estabelecido em 1998 visa assegurar que sejam oferecidos todos os meios, métodos e técnicas disponíveis para a regulação da fecundidade através de ações preventivas e educativas e pela garantia de oferta igualitária à informação, assegurando o direito que toda pessoa tem à assistência especializada e acesso aos recursos que permitam optar livre e conscientemente pela maternidade ${ }^{1}$

A gravidez não planejada constitui um problema de saúde pública, a ocorrência está associada ao uso inadequado dos métodos contraceptivos ou até mesmo ausência de utilização dos métodos e, fatores como baixa escolaridade, dependência financeira de parceiros ou familiares, bem como por deficiência de ações educativas realizadas no planejamento familiar ${ }^{2}$.

Dados publicados pelo Instituto Guttmacher em 2017 mostraram que nos países em desenvolvimento, dos estimados 206 milhões de gestações, $43 \%$ não são planejadas, a incidência de gravidez não planejada eleva o risco de morte materna em decorrência de abortos inseguros, isto posto, as ações do planejamento familiar contribuem para redução do número de abortamento provocado ${ }^{3-4}$.

Para a efetivação dos direitos reprodutivos o programa de planejamento familiar baseia-se em ações preventivas e educativas, oferta de informações, meios, métodos e técnicas para regulação da fecundidade, para que a mulher consiga escolher com clareza qual método contraceptivo utilizar, bem como controlar sua sexualidade e capacidade reprodutiva de forma a exercer autonomia na contracepção, esterilização, concepção, aborto, parto e puerpério ${ }^{5}$.

As ações de educação em saúde devem ser realizadas individualmente e em grupo dentro da Estratégia Saúde de Família e fora dela, de forma a possibilitar que as mulheres contribuam umas com as outras no processo de empoderamento feminino, em conseguinte, consigam exercer autonomia nas escolhas quanto à maternidade, bem como evitar a gravidez não planejada, tendo em vista que a educação em saúde promove a qualidade de vida de indivíduos, famílias e comunidades através de associação entre os saberes populares, técnicos e científicos 6 . Desta forma, este estudo teve por objetivo analisar o conhecimento produzido na literatura científica sobre fatores que contribuem para a gravidez não planejada em usuárias do programa de planejamento familiar.

\section{Metodologia}

Foi realizada uma revisão de literatura integrativa, este tipo de estudo é considerado um instrumento indispensável no campo da saúde, pois busca reunir e sintetizar as pesquisas disponíveis referentes ao assunto estudado e permite a fundamentação do conhecimento científico produzido de modo que seja utilizado na prática ${ }^{7}$.

O presente estudo seguiu as etapas propostas por Souza et al. ${ }^{8}$ que correspondem respectivamente, a escolha do tema, definição da pergunta norteadora, definição dos objetivos, escolha dos critérios de inclusão e exclusão, busca na literatura, interpretação dos artigos sendo expressos em resultados e discussão, e por fim, síntese e apresentação do conhecimento. 0 estudo teve como questão norteadora: quais os conhecimentos produzidos na literatura científica sobre os fatores que contribuem para a gravidez não planejada em usuárias do programa de planejamento familiar?

Os critérios utilizados para a inclusão dos artigos foram: artigos que contivessem em seus títulos e/ou resumos os seguintes descritores pesquisados no Descritores em Ciência da Saúde (DeCS): gravidez não planejada; planejamento familiar; e que tivessem textos completos, idioma em português, publicados no período de 2010 a 2018. Os descritores foram combinados da seguinte forma: Planejamento Familiar e Gravidez não Planejada.

Os critérios de exclusão foram: artigos repetidos nas plataformas de dados, arquivos não acessíveis na íntegra, que não se enquadraram no recorte temporal estabelecido e não respondia a questão norteadora da pesquisa.

Para o levantamento dos artigos realizou-se uma busca nas seguintes bases de dados: Literatura Latino-Americana e do Caribe em Ciências da Saúde (LILACS), Base de Dados em Enfermagem (BDENF), Medical Li terature Analysis and Retriveval System Online (MEDLINE) e Scientific Eletronic Library Online (SciELO), a 
busca ocorreu no período de agosto a novembro de 2018.

Foram identificados 24 artigos, após leitura exploratória dos títulos e resumos, excluiu-se 16 artigos, sendo 6 duplicados e 10 excluídos por não atenderem aos critérios de inclusão da pesquisa. Foram incluídos no total de oito artigos, destes, quatro encontravam-se na base de dados LILACS, um no SciELO, um na BIREME e dois na BDENF.

Para a análise dos artigos utilizou-se as variáveis autor, ano de publicação, título do artigo, metodologia, objetivo do estudo, fatores que contribuem para a gravidez não planejada e conclusão do estudo.

\section{Resultados}

Dos oito artigos selecionados para esta revisão, nota-se que todos foram de autoria múltipla, a maioria foi do tipo de estudo corte transversal, um quantitativo exploratório e um documental quantitativo-descritivo. Respondendo a questão norteadora deste estudo, os artigos referem respectivamente que a baixa adesão ao programa de planejamento familiar ${ }^{11,12,14}$, 15, a adolescência $^{9-11}$, ter companheiro fixo ${ }^{2}, 12,13$, o número de gestações ${ }^{10,15}$, não ter companheiro ${ }^{9}$, ${ }^{10}$, possuir baixa renda familiar ${ }^{2,10}$, possuir cor da pele preta ou parda ${ }^{9}$ e possuir baixa escolaridade ${ }^{11}$ são fatores que contribuem para a ocorrência de gravidez não planejada.

Quadro 1: Síntese dos estudos que abordam a relação entre os fatores que contribuem para a gestação não planejada

\begin{tabular}{|c|c|c|c|c|}
\hline Autor (Ano) & Tipo de estudo & Objetivo & $\begin{array}{lr}\text { Fatores } & \text { que } \\
\text { contribuem } & \text { para } \\
\text { gravidez } & \text { não } \\
\text { planejada. } & \end{array}$ & Conclusão \\
\hline $\begin{array}{l}\text { 9. CAMINHA } \\
\text { et al., } 2010 .\end{array}$ & $\begin{array}{l}\text { Corte transversal } \\
\text { descritivo }\end{array}$ & $\begin{array}{l}\text { Observar } r \\
\text { frequência e os } \\
\text { fatores de risco } \\
\text { para a gravidez não } \\
\text { planejada e não } \\
\begin{array}{l}\text { desejada entre } \\
\text { adolescentes. }\end{array}\end{array}$ & $\begin{array}{l}\text { Adolescência } \\
\text { Não } \\
\text { companheiro }\end{array}$ & $\begin{array}{l}\text { Apesar do conhecimento } \\
\text { sobre o uso dos } \\
\text { anticoncepcionais, a alta taxa } \\
\text { de gravidez não planejada } \\
\text { evidencia a necessidade de } \\
\text { políticas públicas mais } \\
\text { efetivas para as adolescentes } \\
\text { nas ESF. }\end{array}$ \\
\hline $\begin{array}{l}10 . \\
\text { PRIETSCH et } \\
\text { al., } 2011 .\end{array}$ & Corte transversal & $\begin{array}{lr}\text { Avaliar o efeito de } \\
\text { possíveis } & \text { fatores } \\
\text { associados } & \text { à } \\
\text { ocorrência } & \text { de } \\
\text { gravidez } & \text { não } \\
\text { planejada } & \text { no } \\
\text { Município de } & \text { Rio } \\
\text { Grande (RS) } & \end{array}$ & $\begin{array}{l}\text { Cor da pele- preta / } \\
\text { parda } \\
\text { Não } \\
\text { companheiro. } \\
\text { Adolescência } \\
\text { Baixa renda familiar. } \\
\text { Aglomeração } \\
\text { familiar. } \\
\text { Número } \\
\text { gestações. de }\end{array}$ & $\begin{array}{l}\text { O programa de } \\
\text { planejamento familiar e a } \\
\text { distribuição de métodos } \\
\text { contraceptivos não estão } \\
\text { sendo eficiente na redução } \\
\text { da gravidez não planejada. }\end{array}$ \\
\hline $\begin{array}{l}\text { 2. COELHO } \\
\text { et al., } 2012\end{array}$ & $\begin{array}{l}\text { Quantitativo } \\
\text { exploratório } \\
\text { transversal. }\end{array}$ & $\begin{array}{l}\text { Estimar } r \text { a } \\
\text { prevalência de } \\
\text { gravidez não } \\
\text { planejada e verificar } \\
\text { a associação com } \\
\text { fatores } \\
\text { sociodemográficos } \\
\text { de mulheres } \\
\text { cadastradas na ESF } \\
\text { em salvador- BA. } \\
\end{array}$ & $\begin{array}{l}\text { Ter companheiro } \\
\text { fixo } \\
\text { Situação } \\
\text { ocupacional. } \\
\text { Baixa renda familiar. }\end{array}$ & $\begin{array}{l}\text { A baixa escolaridade, baixa } \\
\text { remuneração e dependência } \\
\text { financeira do parceiro } \\
\text { contribuem para a } \\
\text { ocorrência da gravidez não } \\
\text { planejada. }\end{array}$ \\
\hline $\begin{array}{l}11 . \\
\text { HONORI- } \\
\text { FRANÇA et }\end{array}$ & Corte Transversal & $\begin{array}{l}\text { Avaliar o perfil de } \\
\text { gestantes } \\
\text { adolescentes }\end{array}$ & $\begin{array}{l}\text { Baixa adesão } \\
\text { planejamento } \\
\text { reprodutivo. }\end{array}$ & $\begin{array}{l}\text { O ato índice de gestação não } \\
\text { planejada entre mulheres } \\
\text { jovens e adolescentes }\end{array}$ \\
\hline
\end{tabular}

Continua... 


\begin{tabular}{|c|c|c|c|c|}
\hline al., 2013 & & $\begin{array}{lr}\text { atendidas } & \text { no } \\
\text { programa e saúde } \\
\text { da família (PSF) } 301 \\
\text { da cidade de } \\
\text { Aragarças, GO. }\end{array}$ & $\begin{array}{l}\text { Adolescência } \\
\text { Baixa escolaridade }\end{array}$ & $\begin{array}{l}\text { usuárias da ESF aponta para } \\
\text { uma ineficiência do } \\
\text { planejamento familiar. }\end{array}$ \\
\hline $\begin{array}{l}12 . \\
\text { EVANGELIST } \\
\text { A et al., } \\
2015 .\end{array}$ & Corte transversal & 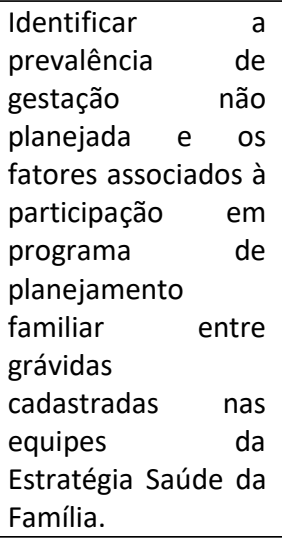 & $\begin{array}{l}\text { Baixa adesão ao } \\
\text { programa de } \\
\text { planejamento } \\
\text { familiar. } \\
\text { Baixa } \\
\text { disponibilidade do } \\
\text { método } \\
\text { anticoncepcional na } \\
\text { ESF. } \\
\text { Ter companheiro } \\
\text { fixo. }\end{array}$ & $\begin{array}{l}\text { A prevalência de gestação } \\
\text { não planeada aponta para a } \\
\text { baixa adesão e baixa } \\
\text { eficiência do programa de } \\
\text { planejamento familiar. }\end{array}$ \\
\hline $\begin{array}{l}13 . \\
\text { PARCERO et } \\
\text { al., } 2017\end{array}$ & $\begin{array}{l}\text { Corte transversal } \\
\text { exploratório. }\end{array}$ & $\begin{array}{l}\text { Descrever } \\
\text { características do } \\
\text { relacionamento } \\
\text { com a companheira } \\
\text { na ocorrência de } \\
\text { gravidez não } \\
\text { planejada. }\end{array}$ & $\begin{array}{l}\text { Ter companheiro } \\
\text { fixo. } \\
\text { Tempo de } \\
\text { relacionamento } \\
\text { maior que três anos. }\end{array}$ & $\begin{array}{l}\text { A situação conjugal, } \\
\text { casada/união estável e o } \\
\text { tempo de união apresentam } \\
\text { alta significância estatística } \\
\text { para ocorrência de gravidez } \\
\text { não planejada. }\end{array}$ \\
\hline $\begin{array}{l}\text { 14. SILVA; } \\
\text { NUNES, } \\
2017 .\end{array}$ & $\begin{array}{l}\text { Documental } \\
\text { quantitativo- } \\
\text { descritivo. }\end{array}$ & $\begin{array}{l}\text { Descrever as } \\
\text { características } \\
\text { reprodutivas, } \\
\text { socioeconômicas e } \\
\text { demográficas de } \\
\text { mulheres na faixa } \\
\text { etária de } 15 \text { a } 49 \\
\text { anos, da equipe } 023 \\
\text { na USF Laurides de } \\
\text { Lima Milhomem. }\end{array}$ & $\begin{array}{ll}\text { Baixa adesão } & \text { ao } \\
\text { programa } & \text { de } \\
\text { planejamento } & \\
\text { familiar. } & \end{array}$ & $\begin{array}{l}\text { As informações passadas } \\
\text { através do planejamento } \\
\text { familiar sobre os métodos } \\
\text { contraceptivos disponíveis } \\
\text { ainda não são suficientes } \\
\text { para reduzir a incidência de } \\
\text { gravidez não planejada }\end{array}$ \\
\hline $\begin{array}{l}\text { 15. BONATTI } \\
\text { et al., } 2018\end{array}$ & $\begin{array}{l}\text { Quantitativo } \\
\text { exploratório } \\
\text { transversal. }\end{array}$ & 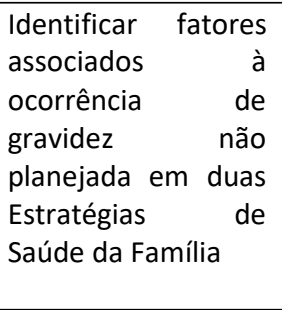 & $\begin{array}{ll}\text { Baixa adesão } & \text { ao } \\
\text { programa } & \text { de } \\
\text { planejamento } & \\
\text { familiar. } & \\
\text { Número } & \text { de } \\
\text { gestações. } & \end{array}$ & $\begin{array}{l}\text { A elevada ocorrência de } \\
\text { gravidez não planejada, } \\
\text { sobretudo entre aquelas que } \\
\text { apresentaram intercorrência } \\
\text { indica a necessidade de } \\
\text { estabelecerem estratégias de } \\
\text { saúde à atenção desta } \\
\text { população. }\end{array}$ \\
\hline
\end{tabular}

Fonte: o Autor

\section{Discussão}

A ocorrência de gestação sem planejamento associa-se à baixa adesão ao programa de planejamento familiar, estudo publicado por Evangelista et al. $^{12}$ mostrou que $58,9 \%$ das mulheres participantes do estudo não planejaram a gravidez, destas, a maioria $(52,6 \%)$ não participaram do programa, Silva e Nunes ${ }^{14}$ contribuem com esse achado demostrando a alta porcentagem de gravidez não planejada $(75,3 \%)$, resultado similar ao de Honório-França et al. ${ }^{11} \mathrm{e}$ Bonatti et al. ${ }^{15}$, que observaram a porcentagem de $75 \%$ e $75,3 \%$ respectivamente.

Isso se dá pelo fato de que as mulheres alegam não ter conhecimento do programa, não 
ter interesse em participar e não ter disponibilidade de tempo para comparecer à Unidade Básica de Saúde ${ }^{12}$, corroborando Cuervo-Vergara et al. $^{16}$ relatam que a alta prevalência de gestação sem planejamento na Colômbia (57\%) está relacionada ao desconhecimento do serviço de planejamento familiar, para Bonatti et al. ${ }^{15}$ esse índice está vinculado a falta de planejamento reprodutivo. A incidência de gravidez não planejada diminui a adesão ao pré-natal, aumenta as chances de desenvolvimento de depressão pós-parto, além de elevar o risco de morte materna em decorrência de abortos inseguros.

$O$ auto índice de gravidez não planejada na adolescência é um fator que indicativo da necessidade de politicas públicas para essa população, segundo pesquisa realizada pelo Fundo de População das Nações Unidas $(\text { UNFPA })^{17}$ em 2017, correspondente aos períodos de 2006 a 2015, o Brasil tem a sétima maior taxa de gravidez na adolescência da América do Sul, com um índice de 65 gestações para cada 1 mil meninas de 15 a 19 anos.

No entanto, Silva et al. ${ }^{14}$ observaram em seu estudo que as adolescentes em sua maioria (76,92\%) tiveram início de atividade sexual entre os 13 a 15 anos de idade, em consonância, Caminha et al. ${ }^{9}$ demostrou que a gestação não planejada foi mais frequentemente $(83,3 \%)$ entre adolescentes com idade inferior a 14 anos, para Medeiros et al. ${ }^{18}$ a iniciação sexual precoce e desprotegida incorre na gravidez não planejada.

A gravidez na adolescência está vinculada à associação de fatores como o alto índice de evasão escolar, baixo nível de escolaridade e o contexto sociodemográfico que as adolescentes estão inseridas, ademais, Araujo e Nery ${ }^{19}$ demostraram que fatores como não ter parceiro, não ter conhecimento sobre utilização dos métodos contraceptivos, ter desejo de engravidar e não saber utilizar a pílula do dia seguinte também contribuem para a ocorrência de gestação sem planejamento na adolescência e suas consequências ${ }^{20}$.

Nesse sentido, é essencial a abordagem sobre saúde sexual e reprodutiva na adolescência, visto que nesse período surgem as mudanças físicas, psicológicas e sociais, desta forma, os profissionais atuantes na Unidade Básica de Saúde devem realizar ações educativas, através de vínculo estabelecido entre famílias e comunidade, considerando as especificidades das famílias do seu território e as desigualdades de raça, gênero, orientação sexual e classe social, a fim de possibilitar o empoderamento e protagonismo dos adolescentes e a condição de escolha por uma gravidez ${ }^{21,22}$.

Fatores como a evasão escolar e dificuldade de retorno à escola, o adiamento ou comprometimento dos projetos de estudo, o surgimento de conflitos familiares, a dificuldade de estabelecer uma família com plena autonomia financeira e independência dos pais e menor chance de qualificação profissional são implicações para a vida das adolescentes que vivenciam uma gravidez sem planejamento ${ }^{23}$.

Outro fator relevante para a ocorrência de gravidez não planejada é a situação conjugal, estudo realizado na cidade de Salvador - BA, observou-se que $56,4 \%$ das mulheres que não planejaram a gestação tinham companheiro fixo ${ }^{2}$, resultado semelhante ao de Parcero et al. ${ }^{13}$ que observaram a porcentagem de 45,7\%, a estabilidade no relacionamento influencia na continuidade do uso do método contraceptivo, o que deixa as mulheres com essa característica mais propensa a gravidez sem planejamento ${ }^{12}$, no entanto, Prietsch et al. ${ }^{10}$ obtiveram como resultado da sua pesquisa que a maioria $(85,2 \%)$ das mulheres que engravidaram sem planejamento relataram estar solteiras ou não ter parceiros fixos, para eles, esse fator associase ao fato de que a população do estudo foram adolescentes.

Mulheres com histórico de gestações anteriores tendem a engravidar sem planejamento, em estudo realizado no município de Rondonópolis no Mato Grosso, observou-se que a maioria das mulheres que não planejaram a gravidez tinham dois filhos ou mais $(46,4 \%)$, sendo classificadas como multigesta ${ }^{15}$, resultado semelhante ao estudo realizado na cidade de Rio Grande - RS, que identificou que ser multigesta aumenta a probabilidade de gravidez sem planejamento ${ }^{10}$, para Garciola e Sebastiam ${ }^{24}$ esse aumento de probabilidade pode ser um sinal do desajuste entre cuidado materno e infantil e o serviço de planejamento familiar.

A dificuldade de acesso ao serviço de planejamento familiar vivenciada por mulheres de classe econômica baixa, associado à dificuldade de aquisição de métodos contraceptivos, contribuem para que cada vez 
mais, essas mulheres tenham filhos sem planejamento, o que aumenta a dependência financeira aos parceiros e dificulta a qualificação no mercado de trabalho, ser multigesta corelaciona-se com fatores socioeconômicos e baixa escolaridade, para Ogido e Schor ${ }^{25}$ mulheres que tem maior número de filhos tem mais dificuldade para inserção no mercado de trabalho do que as que não tem e isto acontece, principalmente, por falta de uma estrutura de apoio como creches e ate mesmo familiares para cuidar das crianças.

Alguns artigos trouxeram que a baixa renda familiar está diretamente ligada à ocorrência de gravidez não planejada, $55,8 \%$ das mulheres que referiram não ter planejado a gestação tinham renda familiar inferior a um salário mínimo, desta forma, o fator como dependência financeira de seus parceiros ou familiares dificulta a autonomia de escolha da mulher, inferindo assim, maior risco de gravidez não planejada ${ }^{2,10}$, para Borrero et al. ${ }^{26}$ as mulheres de baixa renda familiar tem a falsa percepção de baixa fecundidade e relatam a experiência de sabotagem contraceptiva masculina, isso explica-se devido á dependência financeira.

Outro fator associado à ocorrência de gravidez sem planejamento é a cor da pele, Prietsch et al. ${ }^{10}$ observaram em seu estudo que $63,4 \%$ e $73,3 \%$ das mulheres que tiveram gravidez não planejada eram respectivamente pardas e negras, de igual forma Coelho et al. ${ }^{2}$ demostram que $66,7 \%$ das mulheres que tiveram gravidez sem planejamento eram pardas. Um dos principais fatores que contribuíram para que mulheres negras tivessem gestações não planejadas foi a recusa do companheiro em usar métodos contraceptivos e até frustrarem os esforços contraceptivos das esposas com propósito explícito de promover a gravide ${ }^{27}$.

Uma série de fatores socioeconômicos e sociais afetam as mulheres negras, as condições históricas de inserção social contribuem para um desigual e desfavorável acesso aos serviços básicos, inclusive de saúde, as mulheres negras em grande maioria têm menores condições socioeconômicas, menos acesso à educação, possuem condições de moradias precárias por falta de acesso aos serviços, possuem alta taxa de mortalidade materna e infantil e tem menos acesso aos serviços de saúde quando comparadas a mulheres não negras, o que evidencia a vulnerabilidade dessa população ${ }^{5,28}$.

Os resultados dessa revisão demostraram que os fatores associados à ocorrência de gestação não planejada estão interligados, a baixa adesão ao programa de planejamento familiar, principalmente por mulheres mais jovens, com baixa escolaridade, de cor de pele preta ou parda, multigestas, que relataram ter companheiro, que tiveram baixa renda familiar apresentaram maior incidência de gravidez sem planejamento ${ }^{2,10}$.

A associação entre os diversos fatores que estão associados à ocorrência de gestação não planejada revela a necessidade de estudá-los isoladamente, a fim de que políticas públicas sejam implantadas para minimizar os riscos e feitos de uma gravidez sem planejamento, apesar de ser um problema de saúde pública, há poucos estudos nacionais voltados para pesquisa $e$ diminuição dos autos índices de gravidez não planejada, apenas o programa de planejamento familiar sem políticas públicas voltadas para tais fatores torna-se ineficiente para efetivação do planejamento do momento de gestar.

\section{Conclusão}

Essa revisão possibilitou a análise do conhecimento produzido na literatura científica sobre os fatores que contribuem para a gravidez não planejada em usuárias do programa de planejamento familiar, com isso, pôde-se perceber a necessidade de efetivação de políticas públicas voltadas para mulheres inseridas em diversos contextos, tendo em vista que a mulher é um ser holístico e que a gravidez não planejada não ocorre de forma isolada.

Os autos índices de gravidez não planejada denotam a existência de dificuldades no acesso às ações de saúde sexual e saúde reprodutiva, nesse sentido, os profissionais atuantes na Estratégia de Saúde da Família têm papel fundamental na promoção da saúde reprodutiva, pois eles conhecem os diferentes contextos e necessidade de saúde em que as mulheres estão inseridas, estabelecendo uma relação de mútua confiança que favorece a efetivação da assistência.

Há poucos estudos sobre os fatores associados à gravidez não planejada, sabendo-se 
que a baixa adesão ao planejamento familiar correlaciona-se com outros fatores como adolescência, cor da pele, número de filhos, baixa escolaridade, situação conjugal e baixa renda familiar, evidencia-se a necessidade realização de estudos posteriores para cada fator isoladamente, o que permitirá o desenvolvimento e políticas púbicas específicas, isto porque, percebeu-se que apesar da ampla oferta de métodos contraceptivos no âmbito do Sistema Único de Saúde, a falta de ações específicas de promoção e prevenção à saúde, como base nos diferentes fatores socioeconômicos contribui para a ocorrência de gravidez sem planejamento.

\section{Referências}

1. BRASIL. Ministério da Saúde. Política Nacional de Atenção Integral à Saúde da Mulher: Pincípios e diretrizes, Brasília, 2011 [Acesso 22 out 2018 ]; 82 p. Disponível em: < https://bvsms.saude.gov.br/bvs/publicacoes/poli tica_nacional_mulher_principios_diretrizes.pdf>

2. Coelho EAC, Andrade MLS, Vitoriano LVT, Souza JJ, Silva DO, Gusmão MEN, Nascimento ER, Almeida MS. Associação entre gravidez não planejada e o contexto socioeconômico de mulheres em área da Estratégia e Saúde da Família. Acta Paul enferm, São Paulo [internet]. 2012 [Acesso 10 out 2018]; 25(3): 415-422. Disponível

em:

http://www.scielo.br/scielo.php?script=sci_artte xt\&pid=S0103-21002012000300015.>.

3. GUTTMACHER, I. Adding It Up: Investing in Contraception and Maternal and Newborn Health. Nova York. 2017 [acesso em 10 out 2018]. Disponível em:< https://www.guttmacher.org/fact-sheet/addingit-up -contracepção-mnh-2017>

4. BRASIL. Ministério da Saúde. Atenção ao pré-natal de baixo risco. Brasília: Ministério da Saúde, 2013. (Cadernos de Atenção Básica, 32) [Acesso 11 out 2018]; 318 p . Disponível em: < http://bvsms.saude.gov.br/bvs/publicacoes/aten cao_pre_natal_baixo_risco.pdf>

5. BRASIL. Ministério da Saúde. Temático saúde da população negra. Brasília, (Painel de Indicadores do SUS; v. 7, n. 10), 2016 [Acesso 11 out 2018] p. 82. Disponível em: <https://bvsms.saude.gov.br/bvs/publicacoes/te matico_saude_populacao_negra_v._7.pdf>
6. Flores GT, Landerdahl MC, Cortes LF. Ações de enfermeiras em planejamento reprodutivo na atenção primária à saúde. Revista de Enfermagem da UFSM [internet]. 2017 [Acesso 11 out 2018]; 7(4): 643-655, Disponível em:

<https://periodicos.ufsm.br/reufsm/article/view/ 25777/0>.

7. Soaes CB, Hoga LAK, Peduzzi $M$, Sangaleti C, Yonekura T, Silva DRAD. Revisão integrativa: conceitos e métodos utilizados na enfermegem. Rev Esc Enferm USP [internet]. 2014 [Acesso 10 out 2018]; 48(2): 335-45. Disponível em: <http://www.scielo.br/pdf/reeusp/v48n2/pt_008 0-6234-reeusp-48-02-335.pdf>.

8. Souza MT, Silva DS, Carvalho R. Revisão integrativa: o que é e como fazer. Einstein [internet]. 2010 [Acesso 01 out 2018]; 8(1): 1026. Disponível em: < http://www.scielo.br/pdf/eins/v8n1/pt_16794508-eins-8-1-0102.pdf.>.

9. Caminha NO, Freitas LV, Herculano MMS Castro AK. Gestação na adolescencia: do planejamento ao desejo de engravidar-estudo descritivo. Online Brazilian Jornal Of Nursing [internet]. 2010; [Acesso 10 out 2018]; 9(1). Disponível em: $<$ http://www.objnursing.uff.br/index.php/nursing/ article/view/j.1676-4285.2010.2872/651>.

10. Prietsch SOM, Gonzales-Chica DA, Mendoza-Sasse RA. Gravidez não planejada no extremo Sul do Brasil: Prevalência de fatores associados. Cad. Saúde Pública [internet]. 2011 [Acesso 18 set 2018]; 27(10): 1906-1916. Disponível em: <http://www.scielo.br/scielo.php?pid=S0102311X2011001000004\&script=sci_abstract \&tlng=p $\mathrm{t>}$

11. Honorio-França AC, Cardoso APM, França EL, Ferrari CKB. Gestação precoce e reincidência de gestações em adolescentes e mulheres de uma unidade de estratégia de saúde da família. Rev APS [internet]. 2013 [Acesso 18 out 2018]; 16(2): 129-135. Disponível em:< http://bases.bireme.br/cgi-

bin/wxislind.exe/iah/online/>

12. Evangelista $C B$, Barbieri $M$, Silva PLN. Gravidez não planejada e fatores associados à participação em programa de planejamento familiar. Revista de Pesquisa: Cuidado é Fundamental Online [internet]. 2015 [Acesso 08 
set 2018]; 7(2): 2464-2474. Disponível em:< http://www.seer.unirio.br/index.php/cuidadofun damental/article/view/3633>

13. Parcero SMJ, Coelho EAC, Ameida MS, Almeida MS, Nascimento ER. Características do relacionamento entre a mulher e seu parceiro na acorrência de gravidez não planejada. Rev. baiana enferm [internet]. 2017 [Acesso 10 out 2018]; 31(2): 17332. Disponível em: <https://portalseer.ufba.br/index.php/enfermag em/article/view/17332>

14. Silva JM, Nunes MA. Planejamento familiar: uma base de dados. Revista Online de Pesquisa Cuidado é fundamental [internet]. 2017 [Acesso 25 out 2018]; 9(2): 510-519. Disponível em:<http://www.seer.unirio.br/index.php/cuida dofundamental/article/view/5454>.

15. Bonatti AF, Santos GWS, Ribeiro TAN, Santos DAS, Olinda RA, Oliveira JCS. Fatores Associados ao Tipo de Gestação não Planejada na Estratégia de Saúde da Família. Rev Fund Care Online [internet]. 2018 [Acesso 15 jul 2018]; 10(3): 871-876. Disponível em: <http://www.seer.unirio.br/index.php/cuidadofu ndamental/article/download/6256/pdf_1 >.

16. Cuervo-Vergara AS, Garrido-Gutiérrez JD, Vélez-Álvarez GA, Zuleta-Tobón JJ. Associação entre conhecimento sobre contracepção e gravidez não planejada: estudo transversal. Rev Colomb Obstet Ginecol [internet]. 2015 [Acesso em 08 out 2018]; 66(3): 160-170. Disponível em: <http://www.scielo.org.co/scielo.php?script=sci_ abstract\&pid=S0034-

$74342015000300002 \&$ lng=e\&nrm=iso >.

17. UNFPA. Situação da população mundial: Mundos distantes. UNFPA [internet]. 2017 [Acesso 15 set 2018]. Disponível em:< https://portaldeboaspraticas.iff.fiocruz.br/wpcontent/uploads/2018/01/swop2017.pdf>.

18. Medeiros TFR, Santos SMP, Xavier AG, Gonçalves RL, Mariz SR, Sousa FLP . Vivência de mulheres sobre contracepção na perspectiva de gênero. Rev Gaúcha Enfer [internet]. 2016 [Acesso 21 out 2018]; 37(2) e57350. Disponível em:

<http://www.scielo.br/scielo.php?pid=S1983$14472016000200413 \&$ script=sci_abstract\&tlng=p $t>$

19. Araujo AKL, Nery IS. Conhecimento sobre contracepção e fatores associados ao planejamento da gravidez na adolescência. Revista Cogitare Enfermagem [internet]. 2018
[Acesso 21 out 2018]; 23(2): e 55841. Disponível em:<

https://revistas.ufpr.br/cogitare/article/view/558 41>.

20. Jezo RFV, Ribeiro IKS, Araujo A, Rodrigues BA. Gravidez na adolescência: pefil das gestantes e mães adolescentes em uma nunidade básica de saúde. Revista de Enfermagem do Centro-Oeste Mineiro [internet]. 2017 [Acesso 01 nov 2018]; 7:e1387. Disponível em:< https://periodicos.ufjf.br/index.php/aps/article/v iew/14358/7758>

21. Brasil. Ministério da Saúde. Saúde sexual e reprodutiva. Brasília, 2013. (Cadernos de Atenção Básica, n. 26) [Acesso 10 fev 2021]; 300 p. Disponível em: < http://bvsms.saude.gov.br/bvs/publicacoes/saud e_sexual_saude_reprodutiva.pdf >

22. Santos JS, Andrade RD, Mello DF, Maia MAC. Educação em saúde na adolescência: contribuições da Estratégia Saúde da Família. Revista Soc. Bras. Enferm. Ped [internet]. 2014 [Acesso 10 fev 2021]; 14(1): 20-6. Disponível em: $<\quad$ https://journal.sobep.org.br/wpcontent/uploads/articles_xml/2238-202X-sobepS1676-3793201400004/2238-202X-sobep-S16763793201400004.x65337.pdf>

23. Taborda JÁ, Silva FC, Ulbricht L, Neves EB. Consequências da gravidez na adolescência para as meninas considerando-se as diferenças socioeconômicas entre elas. Cad. Saúde Colet [internet]. 2014 [Acesso em 26 out 2018]; 22(1): 16-24. Disponível em: <http://www.scielo.br/scielo.php?pid=S1414462X2014000100016\&script=sci_abstract $>$.

24. Garciola I, Sebastiam E. Unintended pregnan- cy in the amazon basin of Ecuador: a multilevel analysis. Int J Equity Health [internet]. 2010 [acesso em 20 out 2018]; 9-14. Disponível em:

<https://equityhealthj.biomedcentral.com/article s/10.1186/1475-9276-9-14>.

25. Ogido R, Schor N. A jovem mãe e o mercado de trabalho. Saúde Soc. [internet]. 2012[acesso em 21 out 2018]; 21(4): 1044-1055. Disponível em: <http://www.scielo.br/scielo.php?pid=S0104$12902012000400021 \&$ script=sci_abstract \&tlng=p $\mathrm{t}>$.

26. Borrero S, Nikolajski C, Steinberg JR, Freedman L, Akers AY, Ibrahim S, Schwarz EB. "Acontece simplesmente": Um estudo qualitativo 
explorando as perspectivas das mulheres de baixos rendimentos sobre a intenção e o planeamento da gravidez. Contraception. Author manuscript [internet]. 2015 [Acesso 21 out 2018]; 91(2):150-156. Disponível em: https://doi.org/10.1016/j.contraception.2014.09. 014>.

27. Nikolajski C, Miller E, McCauley H, Akers A, Schwarz EB, Freedman L, Steinberg J, Ibrahim S, Borrero $S$. Race and reproductive coercion: $A$ qualitative assessment. Womens Health Issues [internet]. 2015 [Acesso 21 out 2018]; 25(3): 216-223. Disponível em:< https://www.ncbi.nlm.nih.gov/pmc/articles/PMC 4430345/>.

28. Brasil. Ministério a Saúde. Política Nacional de Saúde Integral da População Negra: uma política para o SUS. Brasilia, 2017 [Acesso 22 out 2018]; 3 ed, 43 p. Dispoível em:< https://bvsms.saude.gov.br/bvs/publicacoes/poli tica_nacional_saude_populacao_negra_3d.pdf $>$.

\section{Endereço para Correspondência}

Faculdade Zacarias de Góes

Rua A, S/N - Loteamento Jardim Grimaldi Valença/BA

CEP.: 45.400-000

rosemeiresanntos@gmail.com

Recebido em 13/08/2019

Aprovado em 13/02/2021

Publicado em 31/03/2021 